taining chemical extracts from broccoli that boost production of protective enzymes in skin and protect from UV-induced erythema and inflammation in humans (20). With respect to cancer prevention, it is worth asking whether systemic CP-31398 might prevent cancer development in patients with Li-Fraumeni syndrome - a rare autosomal dominant hereditary disorder in which patients possess a mutation in the p53 tumor suppressor gene that greatly increases their susceptibility to cancer. The study by Tang et al. (17) reports exciting progress with clinical relevance and, like all outstanding papers, raises interesting questions for future work. Further studies and clinical translation of the findings of Tang et al. may lead to improved ways of preventing and treating UV light-induced skin cancers that afflict millions of people.

Address correspondence to: Wafik S. El-Deiry, Department of Medicine, University of Pennsylvania School of Medicine, 415 Curie Blvd., CRB 437, Philadelphia, Pennsylvania 19104, USA. Phone: (215) 898-9015; Fax: (215) 573-9139; E-mail: wafik@mail.med.upenn.edu.
1. American Cancer Society. 2007. Skin cancer facts. http://www.cancer.org/docroot/PED/content/ ped_7_1_What_You_Need_To_Know_About_ Skin_Cancer.asp?sitearea=PED.

2. Abdulla, F.R., Feldman, S.R., Williford, S.R., Krowchuk, D., and Kaur, M. 2005.Tanning and skin cancer. Pediatr. Dermatol. 22:501-512.

3. Brash, D.E., et al. 1991. A role for sunlight in skin cancer: UV-induced p53 mutations in squamous cell carcinoma. Proc. Natl. Acad. Sci. U. S. A. 88:10124-10128.

4. Queille, S., et al. 2007. Analysis of skin cancer risk factors in immunosuppressed renal transplant patients shows high levels of UV-specific tandem CC to TT mutations of the p53 gene. Carcinogenesis. 28:724-731.

5. Rady, P., Scinicariello, F., Wagner, R.F., Jr., and Tyring, S.K. 1992. p53 mutations in basal cell carcinomas. Cancer Res. 52:3804-3806.

6. Bolshakov, S., et al. 2003. p53 mutations in human aggressive and nonaggressive basal and squamous cell carcinomas. Clin. Cancer Res. 9:228-234.

7. Aszterbaum, M., et al. 1999. Ultraviolet and ionizing radiation enhance the growth of BCCs and trichoblastomas in patched heterozygous knockout mice. Nat. Med. 5:1285-1291.

8. Marx, J. 2007. Recruiting the cell's own guardian for cancer therapy. Science. 315:1211-1213.

9. Velculescu, V.E., and El-Deiry, W.S. 1996. Biological and clinical importance of the p53 tumor suppressor gene. Clin. Chem. 42:858-868.

10. Ananthaswamy, H.N., et al. 1997. Sunlight and skin cancer: inhibition of p53 mutations in UV-irradiated mouse skin by sunscreens. Nat. Med. 3:510-514.

11. Jensen, E.H., Margolin, K.A., and Sondak, V.K. 2005. Melanoma and other skin cancers. In Cancer management: a multidisciplinary approach. R. Pazdur,
L.R. Coia, W.J. Hoskins, and L.D. Wagman, editors. 9th edition. CMP Healthcare Media. San Francisco, California, USA. 531-561.

12. Neville, J.A., Welch, E., and Leffell, D.J. 2007. Management of nonmelanoma skin cancer in 2007. Nat. Clin. Pract. Oncol. 4:462-469.

13. Foster, B.A., Coffey, H.A., Morin, M.J., and Rastinejad, F. 1999. Pharmacological rescue of mutant p53 conformation and function. Science. 286:2507-2510.

14. Wang, W., Takimoto, R., Rastinejad, F., and El-Deiry, W.S. 2003. Stabilization of p53 by CP-31398 inhibits ubiquitination without altering phosphorylation at serine 15 or 20 or MDM2 binding. Mol. Cell. Biol. 23:2171-2181.

15. Takimoto, R., et al. 2002. The mutant p53-conformation modifying drug, CP-31398, can induce apoptosis of human cancer cells and can stabilize wild-type p53 protein. Cancer Biol. Ther. 1:47-55.

16. Rippin, T.M., et al. 2002. Characterization of the p53-rescue drug CP-31398 in vitro and in living cells. Oncogene. 21:2119-2129.

17. Tang, X., et al. 2007. CP-31398 restores mutant p53 tumor suppressor function and inhibits UVBinduced skin carcinogenesis in mice. J. Clin. Invest. 117:3753-3764.

18. Christophorou, M.A., et al. 2006. The pathological response to DNA damage does not contribute to p53-mediated tumour suppression. Nature. 443:214-217.

19. Smalley, K.S., et al. 2007. An organometallic protein kinase inhibitor pharmacologically activates p53 and induces apoptosis in human melanoma cells. Cancer Res. 67:209-217.

20. Talalay, P., et al. 2007. Sulforaphane mobilizes cellular defenses that protect skin against damage by UV radiation. Proc. Natl. Acad. Sci. U. S. A. 104:17500-17505.

\title{
IL-6 involvement in epithelial cancers
}

\author{
Zachary T. Schafer and Joan S. Brugge
}

Department of Cell Biology, Harvard Medical School, Boston, Massachusetts, USA.

\begin{abstract}
In this issue of the JCI, two reports provide intriguing new information on the role of the inflammatory cytokine IL- 6 in breast and lung cancer. The study by Sansone et al. implicates IL-6 in the instigation of malignant properties in breast cancer stem cells (see the related article beginning on page 3988 ). The study by Gao et al. identifies mutant variants of EGFR as inducers of IL-6 in lung adenocarcinomas (see the related article beginning on page 3846). These studies add to our understanding of potential roles for IL-6 in cancer and further motivate investigations of IL-6-targeted chemotherapeutics.
\end{abstract}

IL-6 is a multifunctional cytokine that was originally characterized as a regulator of immune and inflammatory responses; however, elevated expression of IL- 6 has been detected in multiple epithelial tumors (1). IL-6 binds to a heterodimeric receptor,

Nonstandard abbreviations used: CA-IX, carbonic anhydrase IX.

Conflict of interest: The authors have declared that no conflict of interest exists.

Citation for this article: J. Clin. Invest. 117:3660-3663 (2007). doi:10.1172/JCI34237. which contains the ligand-binding IL- $6 \alpha$ chain and the common cytokine receptor signal-transducing subunit gp130. IL-6 receptor engagement leads to activation of the JAK family of tyrosine kinases, which then stimulate multiple pathways involving MAPKs, PI3Ks, STATs, and other signaling proteins (2).

Given the reported involvement of IL- 6 and its downstream targets in the regulation of cell proliferation, survival, and metabolism, it is not surprising that IL-6 signaling has also been implicated in tumorigenesis (3). However, the nature of IL-6's involvement in cancer has been quite controversial, as dichotomous roles for IL- 6 in both tumor-promoting and -suppressive activities have been reported. For example, IL-6 signaling has been linked to both pro- and antiapoptotic activity in breast cancer cells $(4,5)$. Multiple studies have documented high IL- 6 levels in the serum of patients with certain carcinomas (i.e., breast, lung, lymphoma) and have correlated high IL-6 levels with a poor clinical prognosis (2). These data imply an oncogenic role for IL-6; however, lacking is an understanding of the mechanisms governing IL- 6 production in tumors and the biological role of this cytokine in tumorigenesis. Two reports in this issue of the JCI $(6,7)$ advance our understanding of both of these issues and provide a molecular rationale for the development of anti-IL-6 therapeutics (summarized in Figure 1). 


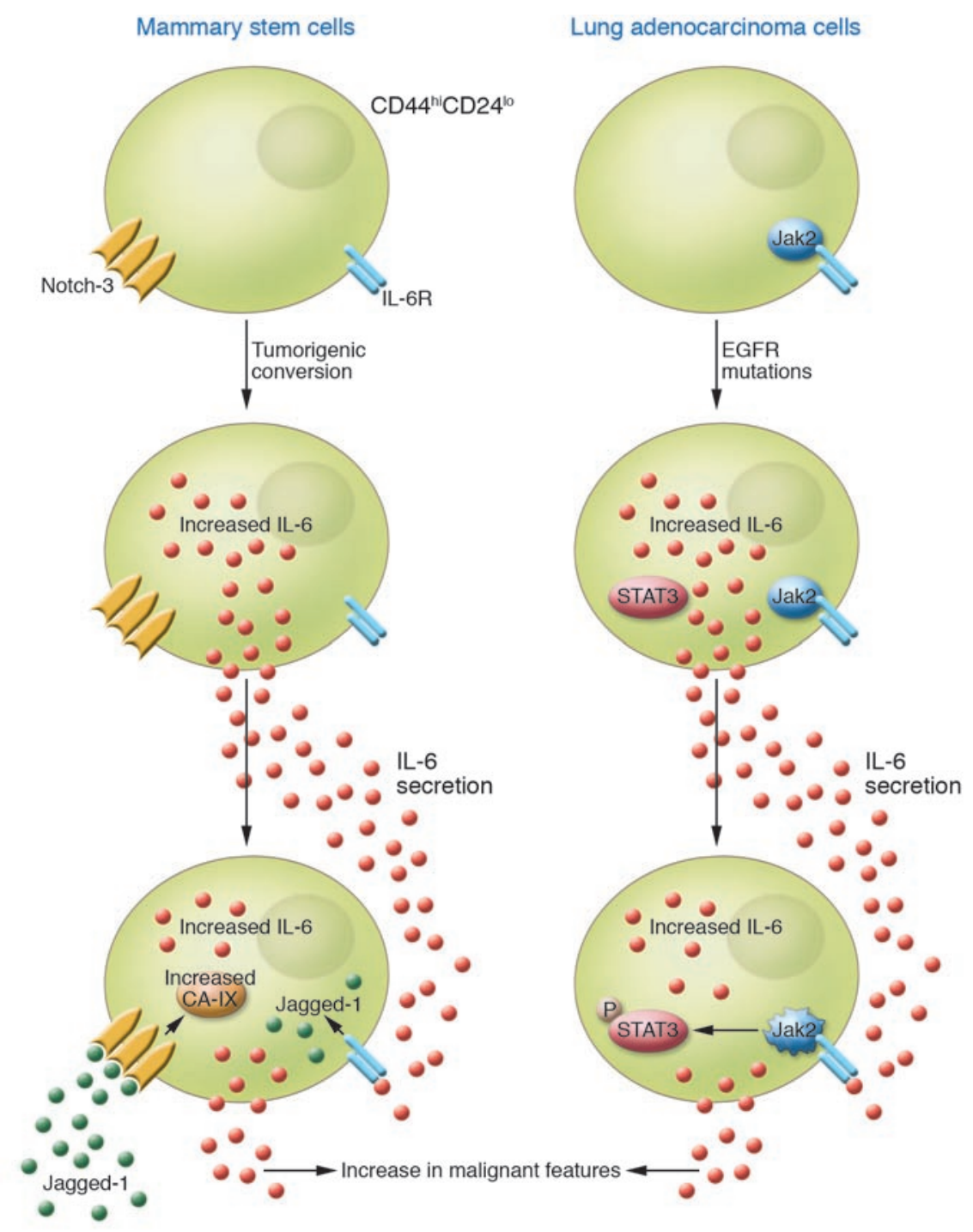

\section{IL-6 production by breast cancer mammospheres}

The identification of putative stem cells in breast tumors has jump-started a rapidly growing field focused on the biology of cancer stem cells in solid tumors (8). The present study by Sansone et al. (6) implicates IL- 6 as a potential regulator of normal and tumor stem cell self renewal. Comparison of mammospheres (multicellular spheroids composed of anchorage-independent self-renewing cells and their derivatives) from normal and tumor tissue from the same patient revealed that IL- 6 mRNA is expressed at significantly higher levels in mammospheres derived from tumor tissue. In addition, spheroids cultured from the MCF-7 breast cancer cell line also contained high IL-6 levels, and treatment with IL-6blocking antibodies suppressed spheroid formation. High expression of IL- 6 was also observed in basal-like breast carcinoma tissues, which are enriched in mammosphere and stem cell markers. The authors further show that IL- 6 can stimulate secondary normal mammospheres and that inhibition of the interaction of IL- 6 with its receptor blunts the size and capacity to form secondary tumor mammospheres, implicating IL-6 as a critical factor in tumor mammosphere self renewal. Sansone et al. (6) further reveal that the ability of IL- 6 to maintain mammosphere self renewal is dependent on the MAPK-dependent upregulation of the transmembrane receptor Notch-3 (a member of the Notch signaling pathway essential for cellular differentiation in a variety of tissues), which has previously been demonstrated to be involved in mammosphere self renewal $(9,10)$. Sansone et al. also describe how IL-6 can upregulate the Notch-3 ligand Jagged-1 to create a positive feedback loop

\section{Figure 1}

Models for role of IL- 6 in breast and lung carcinomas. In this issue of the $\mathrm{JCl}$, Sansone et al. (6) show that the tumorigenic conversion of mammary stem cells (CD44 ${ }^{\text {hi }}$ CD2 $4^{\text {lo }}$ ) results in an increase in IL- 6 expression and secretion. IL- 6 secretion results in a positive feedback loop causing further IL-6 upregulation and secretion. Once secreted, IL-6 can bind the IL-6 receptor (IL-6R), causing the upregulation of the Notch-3 ligand Jagged-1, which triggers the upregulation of CA-IX. While not depicted here, it should be noted that Jagged-1 is a transmembrane ligand. The result of these changes is the promotion of malignant features in these mammary stem cells. Also in this issue, Gao et al. (7) show that EGFR mutations in lung adenocarcinoma cells cause an increase in IL-6 expression and secretion. This, in turn, promotes malignant features in these cells through the IL-6 receptor activation-mediated phosphorylation of STAT3. along the Notch-3/Jagged-1 axis (6). In addition, IL-6 is shown to further promote malignancy in breast cancer stem cells by upregulating the hypoxia response protein carbonic anhydrase IX (CA-IX), which the authors propose permits these cells to survive in hypoxic conditions.

While a role for an immune/inflammatory cytokine like IL-6 in epithelial tumor cells may be unexpected, Sansone et al. (6) propose that stimulation of epithelial stem cells may be part of a natural inflammatory repair program to activate stem cells to replace damaged cells. These studies implicate IL-6 as a critical mediator of mammary stem cell renewal in both normal and tumor contexts.

\section{EGFR-driven IL-6 production in lung tumors}

In a study of non-small-cell lung adenocarcinomas, Gao et al. (7) provide additional 
evidence for the involvement of IL- 6 in cancer and identify an EGFR/IL-6/STAT3 signaling cascade that is important for tumorigenesis. Mutations of the EGFR have been observed in about $10 \%$ of lung adenocarcinomas, and patients whose tumors contain these mutations show increased sensitivity to EGFR tyrosine kinase inhibitors gefitinib (Iressa) or erlotinib (Tarceva) (11, 12). In studies using both mice and human non-small-cell lung adenocarcinoma cell lines, Gao et al. discovered a correlation between activated STAT3 (a downstream target of IL-6) and EGFR mutations in lung tumors (7). Using small hairpin RNA, blocking antibodies, and reconstruction experiments, they demonstrate that activated EGFR induces expression of IL-6, which leads to activation of STAT3. Furthermore, they show that IL-6 expression is substantially elevated in, and IL-6 is secreted by, multiple lung cancer cell lines that harbor EGFR mutations. Gao et al. (7) have characterized a novel mechanism for IL-6 secretion that suggests that anti-IL-6based therapies may have impact in patients with lung adenocarcinomas. Given that approximately $50 \%$ of tumors have activated STAT 3 and only $10 \%$ contain activating EGFR mutations, it is possible that additional alterations may lead to IL-6 secretion and subsequent STAT3 activation. In support of this, a recent report has demonstrated that induction of tumorigenesis in mice by activated Ras, which is mutated in approximately $30 \%$ of human lung adenocarcinomas (13), is dependent on the secretion of IL-6 (14).

The results of Gao et al. (7) nicely complement the work of Sansone et al. (6) by implicating an IL-6 autocrine loop in lung adenocarcinoma. Comparison of the findings in these reports raises obvious questions about the extent to which distinct aspects of each report relate to the other's results. For example, is EGFR involved in IL-6 production in basal breast tumors, as it is in lung tumors? EGFR is enriched in the same breast tumor subclass (basal tumors) that is enriched for IL-6 and Notch-3, so it is plausible that EGFR could regulate IL-6 production in these tumors $(15,16)$. ErbB2, a receptor closely related to EGFR, is amplified in approximately $25 \%$ of breast tumors and activates many of the same pathways as EGFR (12). Therefore, ErbB2 could also stimulate the IL- 6 pathway. While Sansone and coworkers did not examine upstream inducers of IL- 6 production in breast tumors or mammospheres, they did find that IL-6 itself upregulates $I L-6$ mRNA in breast tumor mammospheres, thus creating a positive feedback loop to enhance IL-6 production (6).

Perhaps a more interesting question is: Do lung tumors contain self-renewing cells driven by an IL-6/Notch pathway, as in breast tumors? Gao et al. (7) did not address which IL-6/STAT3 downstream targets are critically involved in lung tumorigenesis; however, the report by Sansone et al. (6) clearly implicates the Notch pathway in stem cell self renewal. Bronchoalveolar stem cells have been identified in normal tissues and lung tumors (17), so it will be of interest to examine involvement of IL- 6 and Notch- 3 in both contexts.

While Sansone et al. (6) focused on the Notch-3 pathway and CA-IX as critical mediators of IL- 6 in breast tumorigenesis, the results of the Gao et al. study implicate the JAK/STAT pathway in lung carcinogenesis (7). It is possible that Notch and JAK/STAT pathways both contribute to IL-6-mediated effects in breast and lung tumors. Indeed, IL- 6 has been shown to activate STAT3 in breast tumor cells lines (18), and ErbB2-induced STAT3 has been shown to regulate tumorigenesis in mouse mammary tumors (19). Crosstalk between these pathways has been reported to occur via facilitation of the interaction of JAK2 and STAT3 by the Notch effectors Hes 1 and Hes5 (20). Thus it would be interesting to determine whether a relationship exists among IL-6, Notch, and JAK/STAT in these tumors.

\section{A future for therapeutic targeting of IL-6 signaling?}

The reports highlighted here $(6,7)$ provide important new insights into potential roles for IL- 6 in epithelial carcinomas and raise the question of whether IL-6-targeted therapies may be effective in treating patients with basal cell breast carcinomas or lung adenocarcinomas carrying EGFR mutations. IL- 6 or IL- 6 receptor antagonists (i.e., CNTO 328, a human-mouse chimeric antibody to human IL-6, and Tocilizumab, humanized anti-IL- 6 receptor antibody) are currently in either phase I or phase II clinical trials in a small subset of cancers and other diseases $(21,22)$. It is difficult to predict the outcome of IL- 6 antagonism in human tumors, because the studies in these reports did not address whether inhibition of IL- 6 would lead to tumor regression or merely prevent expansion of existing tumors that are IL- 6 dependent. It is also unclear whether IL-6 antagonism would provoke tumor cell death. In the absence of tumor cell killing, the opportunity for selection of drug-resistant cells is highly probable. Future studies in mouse tumor models may provide more meaningful predictions of the therapeutic efficacy of IL-6 antagonists. Regardless, these studies strongly implicate IL- 6 in 2 types of epithelial carcinomas and represent significant conceptual advances in our understanding of the role of this cytokine in cancer.

\section{Acknowledgments}

Z.T. Schafer is supported by grant F32CA130327 from the National Cancer Institute. J.S. Brugge is supported by grants from the National Cancer Institute, the Breast Cancer Research Foundation, and Lee Jeans.

Address correspondence to: Joan S. Brugge, Department of Cell Biology, Harvard Medical School, 240 Longwood Avenue, Boston, Massachusetts 02115, USA. Phone: (617) 432-3974; Fax: (617) 432-3969; E-mail: joan_brugge@hms.harvard.edu.

1. Kishimoto, T. 2005. Interleukin-6: from basic science to medicine -40 years in immunology. Annu. Rev. Immunol. 23:1-21.

2. Hong, D.S., Angelo, L.S., and Kurzrock, R. 2007. Interleukin-6 and its receptor in cancer: implications for translational therapeutics. Cancer. doi:10.1002/cncr.22999.

3. Hodge, D.R., Hurt, E.M., and Farrar, W.L. 2005. The role of IL- 6 and STAT3 in inflammation and cancer. Eur. J. Cancer. 41:2502-2512.

4. Chiu, J.J., Sgagias, M.K., and Cowan, K.H. 1996. Interleukin 6 acts as a paracrine growth factor in human mammary carcinoma cell lines. Clin. Cancer Res. 2:215-221.

5. Conze, D., et al. 2001. Autocrine production of interleukin 6 causes multidrug resistance in breast cancer cells. Cancer Res. 61:8851-8858.

6. Sansone, P., et al. 2007. IL-6 triggers malignant features in mammospheres from human ductal breast carcinoma and normal mammary gland. J. Clin. Invest. 117:3988-4002.

7. Gao, S.P., et al. 2007. Mutations in the EGFR kinase domain mediate STAT3 activation via IL- 6 production in human lung adenocarcinomas. J. Clin. Invest. 117:3846-3856.

8. Al-Hajj, M., Wicha, M.S., Benito-Hernandez, A., Morrison, S.J., and Clarke, M.F. 2003. Prospective identification of tumorigenic breast cancer cells. Proc. Natl. Acad. Sci. U. S. A. 100:3983-3988.

9. Sansone, P., et al. 2007. p66Shc/Notch-3 interplay controls self-renewal and hypoxia survival in human stem/progenitor cells of the mammary gland expanded in vitro as mammospheres. Stem Cells. 25:807-815.

10. Dontu, G., et al. 2004. Role of Notch signaling in cell-fate determination of human mammary stem/ progenitor cells. Breast Cancer Res. 6:R605-R615.

11. Paez, J.G., et al. 2004. EGFR mutations in lung cancer: correlation with clinical response to gefitinib therapy. Science. 304:1497-1500.

12. Hynes, N.E., and Lane, H.A. 2005. ERBB receptors and cancer: the complexity of targeted inhibitors. 


\section{commentaries}

Nat. Rev. Cancer. 5:341-354.

13. Slebos, R.J., and Rodenhuis, S. 1992. The ras gene family in human non-small-cell lung cancer. J. Natl. Cancer Inst. Monogr. 1992:23-29.

14. Ancrile, B., Lim, K.H., and Counter, C.M. 2007. Oncogenic Ras-induced secretion of IL6 is required for tumorigenesis. Genes Dev. 21:1714-1719.

15. Farnie, G., et al. 2007. Novel cell culture technique for primary ductal carcinoma in situ: role of Notch and epidermal growth factor receptor signaling pathways. J. Natl. Cancer Inst. 99:616-627.

16. Siziopikou, K.P., and Cobleigh, M. 2007. The basal subtype of breast carcinomas may represent the group of breast tumors that could benefit from EGFR-targeted therapies. Breast. 16:104-107.

17. Kim, C.F., et al. 2005. Identification of bronchioalveolar stem cells in normal lung and lung cancer. Cell. 121:823-835.

18. Berishaj, M., et al. 2007. Stat3 is tyrosinephosphorylated through the interleukin-6/glycoprotein 130/Janus kinase pathway in breast cancer. Breast Cancer Res. 9:R32.

19. Guo, W., et al. 2006. Beta 4 integrin amplifies ErbB2 signaling to promote mammary tumorigenesis.
Cell. 126:489-502

20. Kamakura, S., et al. 2004. Hes binding to STAT3 mediates crosstalk between Notch and JAK-STAT signalling. Nat. Cell Biol. 6:547-554.

21. Zaki, M.H., Nemeth, J.A., and Trikha, M. 2004. CNTO 328, a monoclonal antibody to IL-6, inhibits human tumor-induced cachexia in nude mice. Int. J. Cancer. 111:592-595.

22. Paul-Pletzer, K. 2006. Tocilizumab: blockade of interleukin-6 signaling pathway as a therapeutic strategy for inflammatory disorders. Drugs Today (Barc). 42:559-576. 